\section{Approximation of the inverse normal distribution function}

\author{
ALFRED L. BROPHY \\ Behavioral Science Associates, West Chester, Pennsylvania
}

Hastings (1955, pp. 191-192) developed two approximations of the inverse of the normal distribution function. The more accurate of these approximations (Approximation 68) appears to be the most widely used method of estimating the standard normal deviate (z) corresponding to a given probability (p). This approximation was reprinted in Zelen and Severo (1964, section 26.2.23), and, as noted by Emerson (1979), it has long been available in the IBM Scientific Subroutine Package. However, other approximation methods should be considered. This paper compares Hastings's approximations with seven other compact approximations of the inverse of the normal distribution function. The results complement Brophy's (1983) comparison of approximations of the normal distribution function.

Table 1 shows the approximation methods, in BASIC, together with a short driver program to input a value of $p$, call a selected method, and print the calculated $z$. The first two methods are Hastings's (1955) rational approximations (Approximations 67 and 68). The third is a similar approximation by Hill and Davis (1973). It is one of seven equations designed to supply an initial estimate in a more elaborate algorithm, and it is the most accurate of the seven when used independently. The fourth method, by Odeh and Evans (1974), is another rational approximation, but it uses higher order polynomials than the preceding methods. The fifth method, developed by Beasley and Springer (1977), comprises two rational approximations, one for $.08 \leq p \leq .92$ and the other for more extreme $p$ values. The sixth method, which seems not to have been suggested elsewhere, applies Beasley and Springer's tail approximation to all values of $p$. (The coefficients given in Table 1 for Beasley and Springer's approximations are rounded from their original 11- and 12-figure values.) The seventh method, proposed by Bailey (1981), consists of another pair of approximations, one for $.0000022 \leq p$ $\leq .9999978$ and the other for more extreme $p$ values. The crossover point between the approximations, which differs slightly from that suggested by Bailey, was established empirically. The eighth method applies Bailey's approximation for the central part of the distribution to all values of $p$. The final method is based on Koehler's (1983) approximation of two-sided critical values of the $t$ distribution, with adaptation for infinite degrees of freedom and one-tailed $\mathrm{p}$.

The author's mailing address is: Behavioral Science Associates, P.O. Box 748, West Chester, PA 19381.
Tests of the Approximations. Tests of the approximations were run in single precision on a TRS-80 Model I computer. Each approximation was tested with 31 onetailed p values: $.005, .01, .025, .05$ through .50 in increments of .05 , and $10^{-n}$, where $n$ signifies all integers from 3 through 20 . Correct values of $z$ corresponding to these p values were taken from White's (1970) 20-decimal tables. Before using Odeh and Evans's (1974) approximation, all coefficients were rounded to seven significant figures.

The accuracy of each approximation was assessed in terms of its maximum absolute error in calculated $\mathrm{z}$ for the $p$ values tested. Table 2 summarizes the results of the tests. In each of the first four approximation methods, the maximum absolute error for $10^{-20} \leq p<.0001$ differs from the maximum absolute error for $.0001 \leq p \leq .5$ by less than .00005 , demonstrating remarkable success in maintaining precision in the extreme tail of the distribution.

The most accurate of the approximations is that of Odeh and Evans (1974). As tested, it is accurate to five decimal places, but greater accuracy can be obtained by evaluating the approximation in double precision or on a computer with standard numeric precision greater than the Model I's six digits. For example, Odeh and Evans reported seven-decimal accuracy using double precision on an IBM 370/145, and supplementary tests for the present study with 10-figure coefficients found equivalent accuracy on a Radio Shack Color Computer, which has nine-digit precision.

The maximum absolute error obtained with Hastings's (1955) Approximation 68 was close to the .00045 usually reported for that approximation (Zelen \& Severo, 1964, section 2.26.23). The Hill and Davis (1973) approximation was somewhat more accurate, slightly faster, and shorter, so it is a viable alternative to Approximation 68 . Beasley and Springer's (1977) tail approximation also is an attractive alternative if accuracy in the extreme tail of the distribution is not required. Its maximum absolute error for $10^{-13} \leq \mathrm{p} \leq .5$ is lower than that of either Approximation 68 or Hill and Davis's approximation.

Even the relatively inaccurate Koehler (1983) routine may be satisfactory for some purposes. Consider the transformation of a percentile (C) to a Wechsler-type deviation IQ score by the statement IQ $=\operatorname{INT}(100.5+15 * Z)$, where $\mathrm{Z}$ is an approximated normal deviate obtained by inputting $\mathrm{p}=1-(\mathrm{C} / 100)$. Koehler's approximation yields the same IQ as Odeh and Evans' (1974) for all but four of the percentiles between 1 and 99; the exceptions are incorrect by only one IQ point. Nevertheless, the Koehler routine offers little advantage over Hastings's (1955) Approximation 67, which is substantially more accurate.

Other Approximations. If greater accuracy is desired than is provided by the Odeh and Evans (1974) approximation, a more complex procedure can be used. An initial 
Table 1

Nine Approximations of the Inverse Normal Distribution Function

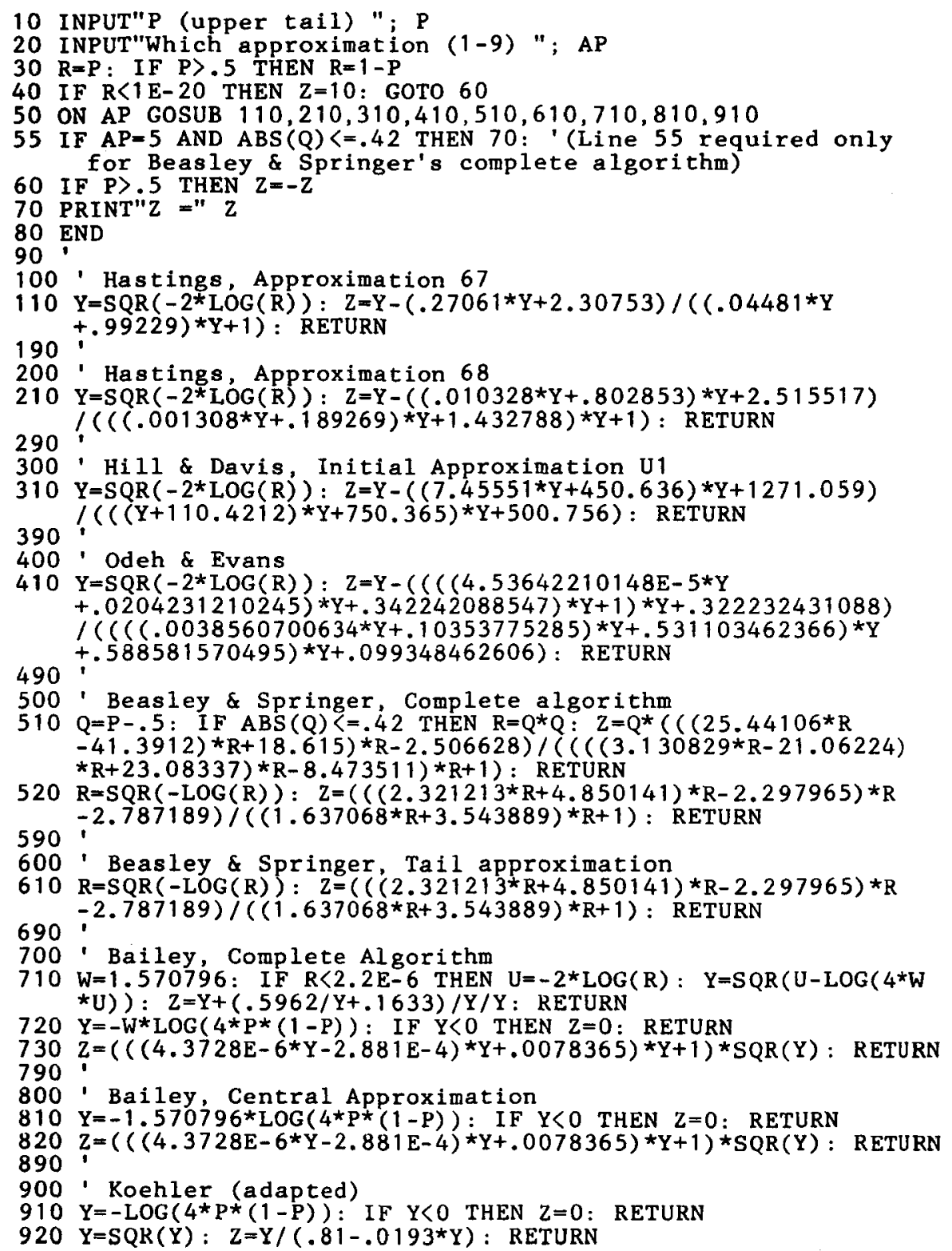

estimate $\left(z^{\prime}\right)$ from one of the approximations listed in Table 1 or from another Hill and Davis (1973) equation can be improved by the Newton-Raphson method (e.g., Milton \& Hotchkiss, 1969) or by a Taylor series (e.g., Cunningham, 1969; Hill \& Davis, 1973) after calculation of the $p$ corresponding to $z^{\prime}$ by an approximation of the normal distribution function (see Brophy, 1983). Although highly precise results can be obtained by these methods, the routines are longer and slower than those tested in the present study, and their precision is unnecessary for most practical purposes.

Hamaker (1978), Page (1977), and Schmeiser (1979) developed very simple approximations that are convenient for use with hand calculators. Each of these is more accurate than Koehler (1983) for some values of $p$, but each has a maximum error greater than that of Hastings's (1955) Approximation 67. Therefore, they are not presented here.

Language and Modifications. The approximations are written in TRS-80 Level II BASIC, a version of Microsoft BASIC. Little or no modification is required for other BASIC dialects, and translation into FORTRAN is relatively simple.

If $p$ is very close to unity, loss of precision may occur 
Table 2

Accuracy for Two Ranges of Probability, Mean Execution Time (in Seconds), and Length (in Bytes) of Nine Approximations of the Inverse Normal Distribution Function, Calculated on the TRS-80 Model I Computer

\begin{tabular}{|c|c|c|c|c|}
\hline \multirow[b]{2}{*}{ Approximation } & \multicolumn{2}{|c|}{ Maximum Absolute Error } & \multirow[b]{2}{*}{ Time } & \multirow[b]{2}{*}{ Length } \\
\hline & $.0001 \leq p \leq .5$ & $10^{-20} \leq \mathrm{p}<.0001$ & & \\
\hline \multicolumn{5}{|l|}{ Hastings } \\
\hline Approximation 67 & .00277 & .00281 & .4 & 68 \\
\hline Approximation 68 & .00044 & .00044 & .5 & 97 \\
\hline Hill \& Davis & .00035 & .00035 & .4 & 95 \\
\hline Odeh \& Evans & .000001 & .000002 & 8 & $146^{*}$ \\
\hline \multicolumn{5}{|l|}{ Beasley \& Springer } \\
\hline Complete algorithm & .000001 & $.00084 * *$ & 6 & 236 \\
\hline Tail approximation & .00020 & $.00084 * *$ & .5 & 97 \\
\hline \multicolumn{5}{|l|}{ Bailey } \\
\hline Complete algorithm & .00027 & .00014 & .5 & 178 \\
\hline Central approximation & .00027 & 4.49003 & .5 & 101 \\
\hline Koehler (adapted) & .01913 & .55129 & .3 & 69 \\
\hline
\end{tabular}

in evaluating $(1-p)$. In such cases, it is advisable to input the area of the smaller tail directly rather than the larger upper-tail $p$. If the lower-tail $p$ is input, the inequality sign in Line 60 of the driver program should be reversed so that $\mathrm{z}$ will be negative when $\mathrm{p}<.5$.

Availability. A listing of the approximations can be obtained without charge from the author.

\section{REFERENCES}

BaIley, B. J. R. (1981). Alternatives to Hastings' approximation to the inverse of the normal cumulative distribution function. Applied Statistics, 30, 275-276.

BeAsley, J. D., \& SPRINGER, S. G. (1977). Algorithm AS 111: The percentage points of the normal distribution. Applied Statistics, 26, 118-121.

BROPHY, A. L. (1983). Accuracy and speed of seven approximations of the normal distribution function. Behavior Research Methods \& Instrumentation, 15, 604-605.

Cunningham, S. W. (1969). Algorithm AS 24: From normal integral to deviate. Applied Statistics, 18, 290-293.

EMERSON, P. L. (1979). Computer approximation of the inverse of the normal distribution function. Behavior Research Methods \& Instrumentation, 11, 397-398.

HAMAKER, H. C. (1978). Approximating the cumulative normal distribution and its inverse. Applied Statistics, 27, 76-77.
Hastings, C., JR. (1955). Approximations for digital computers. Princeton, NJ: Princeton University Press.

HILl, G. W., \& Davis, A. W. (1973). Algorithm 442: Normal deviate. Communications of the ACM, 16, 51-52.

KOEHLER, K. J. (1983). A simple approximation for the percentiles of the $t$ distribution. Technometrics, 25, 103-105.

Milton, R. C., \& Hotchkiss, R. (1969). Computer evaluation of the normal and inverse normal distribution functions. Technometrics, 11, 817-822.

ODEH, R. E., \& Evans, J. O. (1974). Algorithm AS 70: The percentage points of the normal distribution. Applied Statistics, 23, 96-97.

PAGE, E. (1977). Approximations to the cumulative normal function and its inverse for use on a pocket calculator. Applied Statistics, 26, 75-76.

SCHMEISER, B. W. (1979). Approximations to the inverse cumulative normal function for use on hand calculators. Applied Statistics, 28, 175-176.

WhITE, J. S. (1970). Tables of normal percentile points. Journal of the American Statistical Association, 65, 635-638.

Zelen, M., \& Severo, N. C. (1964). Probability functions. In M. Abramowitz \& I. A. Stegun (Eds.), Handbook of mathematical functions (pp. 925-995). Washington, DC: U.S. Government Printing Office.

(Revision accepted for publication February 26, 1985.) 